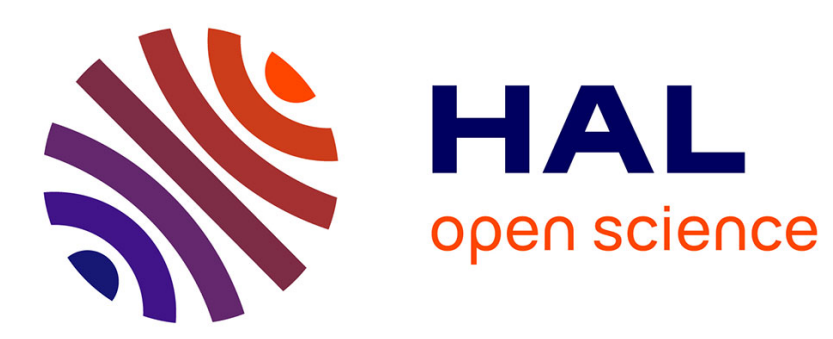

\title{
Interactions and charge fractionalization in an electronic Hong-Ou-Mandel interferometer
}

\author{
Claire Wahl, Jérôme Rech, Thibaut Jonckheere, Thierry Martin
}

\section{To cite this version:}

Claire Wahl, Jérôme Rech, Thibaut Jonckheere, Thierry Martin. Interactions and charge fractionalization in an electronic Hong-Ou-Mandel interferometer. Physical Review Letters, 2014, 112 (4), pp.046802. 10.1103/PhysRevLett.112.046802 . hal-00846907

\section{HAL Id: hal-00846907 https://hal.science/hal-00846907}

Submitted on 30 Nov 2020

HAL is a multi-disciplinary open access archive for the deposit and dissemination of scientific research documents, whether they are published or not. The documents may come from teaching and research institutions in France or abroad, or from public or private research centers.
L'archive ouverte pluridisciplinaire HAL, est destinée au dépôt et à la diffusion de documents scientifiques de niveau recherche, publiés ou non, émanant des établissements d'enseignement et de recherche français ou étrangers, des laboratoires publics ou privés. 


\title{
Interactions and Charge Fractionalization in an Electronic Hong-Ou-Mandel Interferometer
}

\author{
Claire Wahl, ${ }^{1,2, *}$ Jérôme Rech, ${ }^{1,2}$ Thibaut Jonckheere, ${ }^{1,2}$ and Thierry Martin ${ }^{1,2}$ \\ ${ }^{1}$ Aix Marseille Université, CNRS, CPT, UMR 7332, 13288 Marseille, France \\ ${ }^{2}$ Université de Toulon, CNRS, CPT, UMR 7332, 83957 La Garde, France
}

(Received 19 July 2013; published 27 January 2014)

\begin{abstract}
We consider an electronic analog of the Hong-Ou-Mandel (HOM) interferometer, where two single electrons travel along opposite chiral edge states and collide at a quantum point contact. Studying the current noise, we show that because of interactions between copropagating edge states, the degree of indistinguishability between the two electron wave packets is dramatically reduced, leading to reduced contrast for the HOM signal. This decoherence phenomenon strongly depends on the energy resolution of the packets. Insofar as interactions cause charge fractionalization, we show that charge and neutral modes interfere with each other, leading to satellite dips or peaks in the current noise. Our calculations explain recent experimental results [E. Bocquillon, et al., Science 339, 1054 (2013)] where an electronic HOM signal with reduced contrast was observed.
\end{abstract}

DOI: 10.1103/PhysRevLett.112.046802

Electron quantum optics aims at transposing quantum optics experiments—-such as Hanbury Brown-Twiss [1,2] (HBT) or Hong-Ou-Mandel [3,4] (HOM) setups-for the manipulation and measurement of single electrons propagating in quantum channels. Electrons differ from photons because of their statistics, the presence of the Fermi sea (FS) in condensed matter systems and the fact that they interact. If controlling a single photon at a time was mastered long ago [5], the emission of single electrons has only been achieved recently [6-11]. This now allows us to implement in the integer quantum Hall effect (QHE) regime the electronic analog of the HOM experiment [12], which, in optics, measures the degree of indistinguishability between two photons colliding on a beam splitter. Here, two electrons propagate along opposite edge states and collide at the location of a quantum point contact (QPC). Several theoretical works [13-17] have addressed the outcome of this experiment at the single electron level (taking full account of the statistics): the modulus of the current correlations at the output of the QHE bar exhibits a dip as a function of the time delay $\delta T$ between injections. When $\delta T=0$, this dip extends down to 0 . When the time delay is large enough, the two electrons no longer interfere and scatter independently at the QPC, and the current correlations correspond to the sum of the two HBT signals.

The puzzle with the recent experiment performed at a filling factor $\nu>1$, is that the HOM dip does not vanish as predicted for $\nu=1$. Here, we provide a theoretical framework for the experiment and we show that the interaction between quantum channels is responsible for the observed effect. Indeed, at $\nu>1$, interactions dramatically change the nature of excitations, leading to energy exchange between the channels and to charge fractionalization [18-27]. Here, we consider a quantum Hall bar at $\nu=2$,
PACS numbers: 73.23.-b, 42.50.-p, 71.10.Pm, 72.70.+m

in the strong coupling regime and at finite temperature $(\Theta \sim 100 \mathrm{mK}$, following the experiment). The effect of interactions between edge states is probed by comparison with previous results obtained at $\nu=1$ without interactions [17].

Charge fractionalization.-On each edge, the two copropagating channels are coupled via Coulomb interaction modeled as a short-range interaction $H_{\text {int }}$. Label $j=1,2$ identifies outer and inner channels while $r=R, L$ stands for right and left moving ones. The electronic annihilation operator at position $x$ and time $t$ reads $\psi_{j, r}(x, t)=$ $U_{r} / \sqrt{2 \pi a} e^{i \phi_{j, r}(x, t)}$ with $\phi$ the chiral Luttinger bosonic field [28], $U_{r}$ a Klein factor, and $a$ a cutoff parameter. The Hamiltonian is the sum of its kinetic and interaction contributions

$$
\begin{gathered}
H_{\text {kin }}=\sum_{j=1,2} v_{j} \frac{\hbar}{\pi} \sum_{r=R, L} \int d x\left(\partial_{x} \phi_{j, r}\right)^{2}, \\
H_{\text {int }}=2 u \frac{\hbar}{\pi} \sum_{r} \int d x\left(\partial_{x} \phi_{1, r}\right)\left(\partial_{x} \phi_{2, r}\right),
\end{gathered}
$$

where $u$ describes the interaction strength. The charge density operator is $q_{j, r}(x, t)=e / \pi \partial_{x} \phi_{j, r}(x, t)$, and thus, Eq. (2) describes a local capacitive coupling between copropagating channels. Intrachannel interactions can be taken into account by a renormalization of the velocities $v_{1,2}$. The full interacting problem can now be diagonalized with a rotation of angle $\theta$ defined as $\tan (2 \theta)=2 u /\left(v_{1}-\right.$ $v_{2}$ ) which expresses the coupling strength. Since the strong coupling regime $\theta=\pi / 4$ seems to be the most relevant experimentally [29], we focus on $v_{1}=v_{2}=v_{F}=1$. The Hamiltonian is next expressed in terms of the rotated fields 
$\phi_{ \pm}=\left(\phi_{2} \pm \phi_{1}\right) / \sqrt{2}$ as $H=\hbar / \pi \sum_{r} \int d x v_{+}\left(\partial_{x} \phi_{+, r}\right)^{2}+$ $v_{-}\left(\partial_{x} \phi_{-, r}\right)^{2}$. It describes the free propagation of the two collective modes: a fast charge mode traveling with velocity $v_{+}=1+u$ and a slow neutral mode propagating at $v_{-}=1-u$. Along a given edge, each of these modes can be viewed as two separate excitations propagating on the different channels composing the edge, and characterized by the charge they carry $(\oplus$ or $\ominus$ ). The single electron source is modeled through the injection of single electronic wave packets along the edges at a given distance from the QPC, which amounts to calculating all average values over a prepared state [17]. In order to be as close as can be to the experiment [12], electrons are injected along the outer channel as exponential wave packets in real space $\varphi_{L}(x)=\sqrt{2 \Gamma} e^{-i \epsilon_{0} x} e^{-\Gamma x} \theta(x)$. The higher the energy resolution $\gamma=\epsilon_{0} / \Gamma$ of the wave packet, the more it decoheres, as its energy distribution is more distorted from its original shape. Propagation in real space simply amounts to translating the charge and neutral modes with their respective velocity even though their energy profile is strongly modified along the edge.

$H B T$.-We partition at a QPC the excitations propagating along the left-moving edge following the injection along the outer channel of an exponential wave packet at position $x=L$. Depending on which counter-propagating channels are connected at the QPC, one can distinguish two setups (see Fig. 1) which we hereby label as setup 1 and 2, corresponding, respectively, to the partitioning of the outer $(s=1)$ or the inner channel $(s=2)$. Both these setups can be realized in practice, and although experimental investigations have focused so far on setup 1 , we will be considering them both.

The quantity of interest is the zero-frequency current correlations $[30,31]$ measured on the partitioned channel:

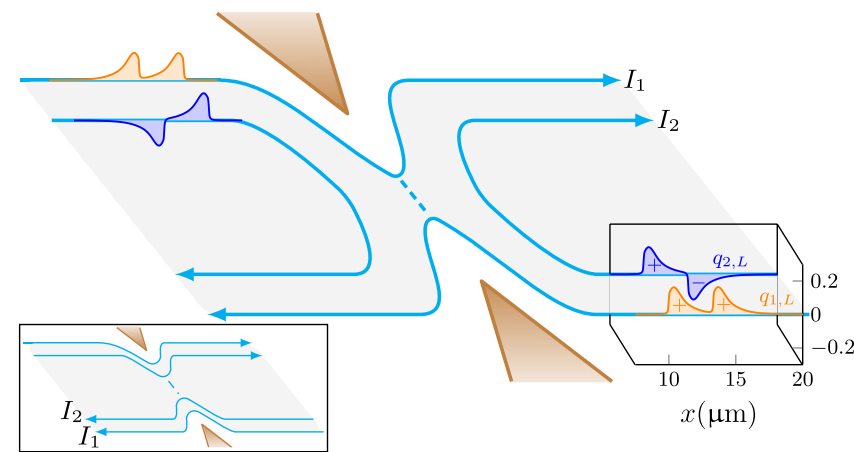

FIG. 1 (color online). The setups: two opposite edge states, each made out of two interacting copropagating channels, meet at a QPC. An electronic wave packet is injected on both incoming outer channels. (Main) Setup 1: backscattering occurs for outer channels. (Left inset) Setup 2: backscattering occurs for inner channels. (Right inset) Electron density as a function of position for an energy resolved packet imaged after propagating on a $5 \mu \mathrm{m}$ length, revealing the presence of two modes composed each of two $\oplus / \ominus$ excitations.
$S_{\mathrm{HBT}}=\int d t d t^{\prime}\left\langle I_{s}(t) I_{s}\left(t^{\prime}\right)\right\rangle-\left\langle I_{s}(t)\right\rangle\left\langle I_{s}\left(t^{\prime}\right)\right\rangle$, where the averages are performed on the prepared state $\left|\phi_{L}\right\rangle=$ $\int d x \varphi_{L}(x) \psi_{1, L}^{\dagger}(x+L, 0)|0\rangle$. All integrals are computed from $-\infty$ to $+\infty$, and the FS contribution has been removed. The linear dispersion of the edges allows us to compute the noise at the immediate output of the QPC, without loss of generality. The latter is described by its scattering matrix which relates the outgoing fields along the partitioned channel to the incoming ones

$$
\left(\begin{array}{l}
\psi_{s, R} \\
\psi_{s, L}
\end{array}\right)^{\text {outgoing }}=\left(\begin{array}{cc}
\sqrt{\mathcal{T}} & i \sqrt{\mathcal{R}} \\
i \sqrt{\mathcal{R}} & \sqrt{\mathcal{T}}
\end{array}\right)\left(\begin{array}{l}
\psi_{s, R} \\
\psi_{s, L}
\end{array}\right)^{\text {incoming }},
$$

where $\mathcal{T}$ and $\mathcal{R}=1-\mathcal{T}$ are the transmission and reflection probabilities. This scattering approach, albeit used for interacting fermion fields, is justified because both the interaction and the tunneling are purely local. The QPC is, thus, not included in the interaction region, and fermions are locally free at this location. The proof, based on refermionization, is given in the Supplemental Material (SM) [32]. Equation (3) allows us to express the noise in terms of the incoming operators only [33]. Since the injection process is noiseless within our model, we are left with

$$
\begin{aligned}
S_{\mathrm{HBT}}= & -e^{2} \mathcal{R} T \int d t d t^{\prime}\left\langle\psi_{s, R}^{\dagger}(t) \psi_{s, R}\left(t^{\prime}\right)\right\rangle\left\langle\psi_{s, L}(t) \psi_{s, L}^{\dagger}\left(t^{\prime}\right)\right\rangle \\
& +\left\langle\psi_{s, L}^{\dagger}(t) \psi_{s, L}\left(t^{\prime}\right)\right\rangle\left\langle\psi_{s, R}(t) \psi_{s, R}^{\dagger}\left(t^{\prime}\right)\right\rangle,
\end{aligned}
$$

where all quantities are computed at the input of the QPC. The averages are expressed in terms of the fast and slow Green's functions of the bosonic fields

$$
\begin{aligned}
S_{\mathrm{HBT}}= & -\frac{2 e^{2} \mathcal{R} T}{(2 \pi a)^{3} \mathcal{N}} \operatorname{Re} \\
& \left\{\int d y_{L} d z_{L} \varphi_{L}\left(y_{L}\right) \varphi_{L}^{*}\left(z_{L}\right) g\left(0, z_{L}-y_{L}\right)\right. \\
& \left.\times \int d t d \tau \operatorname{Re}\left[g(\tau, 0)^{2}\right]\left[\frac{h_{s}\left(t ; y_{L}+L, z_{L}+L\right)}{h_{s}\left(t+\tau ; y_{L}+L, z_{L}+L\right)}-1\right]\right\},
\end{aligned}
$$

where $\mathcal{N}=\left\langle\phi_{L} \mid \phi_{L}\right\rangle, s=1,2$ is the setup considered and

$$
\begin{gathered}
g(t, x)=\left[\frac{\sinh \left(i \frac{\pi a}{\beta v_{+}}\right)}{\sinh \left(\frac{i a+v_{+}+x}{\beta v_{+} / \pi}\right)} \frac{\sinh \left(i \frac{\pi a}{\beta v_{-}}\right)}{\sinh \left(\frac{i a+v_{-}-x}{\beta v_{-} / \pi}\right)}\right]^{1 / 2}, \\
h_{s}(t ; x, y)=\left[\frac{\sinh \left(\frac{i a-v_{+}+t+x}{\beta v_{+} / \pi}\right)}{\sinh \left(\frac{i a+v_{+}+t-y}{\beta v_{+} / \pi}\right)}\right]^{\frac{1}{2}}\left[\frac{\sinh \left(\frac{i a-v_{-} t+x}{\beta v_{-} / \pi}\right)}{\sinh \left(\frac{i a+v_{-} t-y}{\beta v_{-} / \pi}\right)}\right]^{s-\frac{3}{2}} .
\end{gathered}
$$

Numerical integration is handled with a quasi-Monte Carlo algorithm using importance sampling [34] (details are given in the SM [32]). Since the partition noise counts 
the number of particle-hole excitations [16], the absolute value of the noise increases with the interaction strength and the energy resolution of the packet: as a single electron injected above the FS relaxes, it creates particle-hole pairs near the Fermi energy [35] which scatter at the QPC. These spurious excitations are more numerous for an energyresolved packet, resulting in a larger noise. The dependence of the noise on $L$ is then governed by two opposing effects. While the eigenmodes are dragged apart when $L$ rises, the number of particle-hole pairs increases and finally diverges as $\log L$ at zero temperature [36] (see SM [32]), leading to the same divergence in the noise. However, at finite temperature, $S_{\mathrm{HBT}}$ is dramatically reduced because of antibunching with thermal excitations at the output of the QPC [37]. This tends to minimize the contribution to the noise from low-energy quasiparticles, which were dominant at $\Theta=0$. The finite temperature, thus, acts as a low-energy cutoff, washing out the length dependence of the noise, which typically is constant for $L \geq 2 \mu \mathrm{m}$, at $\Theta \sim 100 \mathrm{mK}$.

HOM.-The next step is to make two wave packets collide at the QPC. The prepared state is now $\left|\phi_{R}\right\rangle \otimes\left|\phi_{L}\right\rangle$, where two electrons are injected on the outer channel of the counter-propagating arms. For simplicity, we consider injections at symmetric positions $\pm L$, focusing on the interference between identical wave packets, $\varphi_{R}(x)=\varphi_{L}(-x)$. The expression for the noise, Eq. (5), is modified as

$$
\begin{aligned}
S_{\mathrm{HOM}}(\delta T)= & -\frac{2 e^{2} \mathcal{R} T}{(2 \pi a)^{4} \mathcal{N}} \operatorname{Re}\left\{\int d y_{L} d z_{L} \varphi_{L}\left(y_{L}\right) \varphi_{L}^{*}\left(z_{L}\right) g\left(0, z_{L}-y_{L}\right) \int d y_{R} d z_{R} \varphi_{R}\left(y_{R}\right) \varphi_{R}^{*}\left(z_{R}\right) g\left(0, y_{R}-z_{R}\right)\right. \\
& \left.\times \int d \tau \operatorname{Re}\left[g(\tau, 0)^{2}\right] \int d t\left[\frac{h_{s}\left(t ; y_{L}+L, z_{L}+L\right)}{h_{s}\left(t+\tau ; y_{L}+L, z_{L}+L\right)} \frac{h_{s}\left(t+\tau-\delta T ; L-y_{R}, L-z_{R}\right)}{h_{s}\left(t-\delta T ; L-y_{R}, L-z_{R}\right)}-1\right]\right\} .
\end{aligned}
$$

As the time delay $\delta T$ between the right- and left-moving electron is varied, we find three characteristic signatures in the noise [see Figs. 2(a)-2(b)]. At $\delta T=0$, a central dip appears, with a depth which depends strongly on the injected packet energy resolution, but very little on the actual setup considered. At $\delta T= \pm 2 L u /\left(1-u^{2}\right)$, side structures emerge symmetrically with respect to the central dip, with a depth and shape that is again conditioned by the
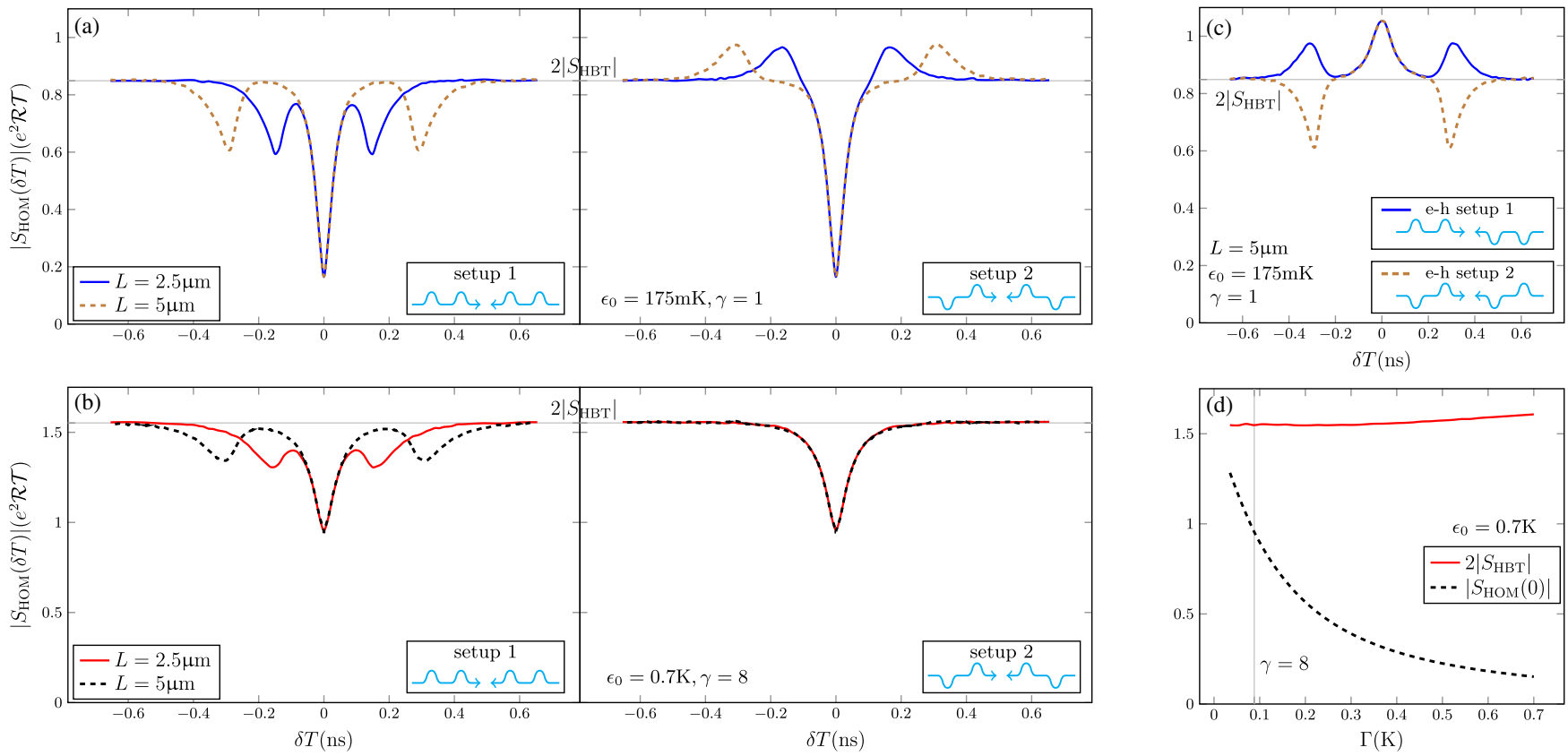

FIG. 2 (color online). (a)-(c) Modulus of $S_{\mathrm{HOM}}$ in units of $e^{2} \mathcal{R} T$ as a function of the time delay $\delta T$, for setups 1 and 2. (a) Packets wide in energy ( $\gamma=1, \epsilon_{0}=175 \mathrm{mK}$ is the injection energy). (b) Energy-resolved packets $(\gamma=8)$ give a lower contrast. Results for setup 1 reveal a triple dip structure, while for setup 2 we obtain a peak-dip-peak structure, with vanishingly small peaks in the case of energyresolved packets. (c) Electron-hole interference: an electron has been injected on the right moving arm and a hole on the left moving one. (d) Modulus of $S_{\mathrm{HOM}}(0)$ and $2 S_{\mathrm{HBT}}$ in units of $e^{2} \mathcal{R} T$ as a function of $\Gamma$, for $\epsilon_{0}=0.7 \mathrm{~K}$. In all plots, $u=0.5$ and $\Theta=0.1 \mathrm{~K}$. 
energy resolution of the wave packet. Interestingly, these results vary critically between setups, as these side structures manifest as dips for setup 1, but peaks for setup 2 . Away from these three features, $S_{\text {HOM }}$ saturates at twice the HBT noise: electrons injected on the two incoming arms scatter independently at the QPC.

This interference pattern is interpreted in terms of the different excitations propagating along the partitioned edge channel. After injection, the electron fractionalizes into two modes. The fast charge mode is composed of two $\oplus$ excitations. The slow neutral mode is made out of a $\oplus$ excitation propagating along the injection channel and a $\ominus$ excitation traveling along the copropagating channel. The central dip, which corresponds to the symmetric situation of synchronized injections, thus, probes the interference of excitations with the same velocity and charge: two fast $\oplus$ excitations then two slow $\oplus$ or $\ominus$ excitations (depending on the setup). These identical excitations interfere destructively, leading to a reduction of the noise (in absolute value), thus, producing a dip. Note that the bottom of this dip is practically insensitive to the chosen setup (and thus, to the partitioned channel) signaling that the interference between identical excitations is independent on the charge they carry.

A striking difference with the $\nu=1$ case is that the central dip never reaches down to 0 , as observed experimentally [12]. The depth of this dip is actually a probing tool of the degree of indistinguishability between the colliding excitations [38]. Our present work suggests that because of the strong interchannel coupling, some coherence is lost in the copropagating channels, and the Coulomb-induced decoherence leads to this characteristic loss of contrast for the HOM dip. This effect gets more pronounced for further energy-resolved packets. As depicted in Figs. 2(a)-2(b), while for "wide" packets in energy $(\gamma=1)$ the contrast is still pretty good, $\eta \sim 0.8$, the loss of contrast can be dramatic for energy-resolved packets, with $\eta \sim 0.4$ for $\gamma=8$. The same trend is observed in experiments [39]. Results for the dip depth at $\Theta=0$ are discussed in the SM [32].

Adjusting $\delta T$ appropriately, one can also probe interferences between excitations that have different velocities. This effect is responsible for the side structures appearing in the noise: at $\delta T=2 L u /\left(1-u^{2}\right)$, the fast right-moving excitation and the slow left-moving one reach the QPC at the same time [40]. In setup 1, these lateral structures correspond to the collision of two $\oplus$ excitations, which interfere destructively, as argued earlier, leading to dips. Their depth is, however, less than half the one of the central dip. This can be attributed to the velocity mismatch between interfering excitations, as it indicates that they are more distinguishable. More interestingly, setup 2 allows us to probe the encounter of excitations with opposite charge (and different velocities), which is expected to lead to constructive interference. This is consistent with the occurrence of lateral peaks in our calculations [see Fig. 2(a), right column].It is reminiscent of what was predicted in the $\nu=1$ case for electron-hole HOM interferometry [17]. The side peaks are more pronounced for small $\gamma$, and become vanishingly small for larger values of the energy resolution, signaling a nontrivial dependence on the packet energy content.

All these lateral dips and peaks are asymmetric as a consequence of the velocity difference between excitations. Typically, the slope is steeper for smaller $|\delta T|$. This asymmetry is similar to the one encountered in the noninteracting $\nu=1$ case for interfering packets with different shapes, where a broad right-moving packet in space collides onto a thin left-moving one [17].

Our approach is general enough to be extended to regimes that have yet to be explored experimentally, such as electron-hole interferometry, where an electron is injected on one edge, while a hole is injected on the other edge [see Fig. 2(c)]. There, we recover three structures in the noise. For both setups, at $\delta T=0 \oplus$ excitations interfere constructively with $\ominus$ excitations, leading to a central peak. However, while setup 1 shows lateral peaks produced by interfering oppositely charged excitations, setup 2 probes the interference of excitations carrying the same charge, leading to lateral dips.

Results concerning the dependence on the energy width of the packet are presented in Fig. 2(d), for an injection well above the FS $\left(\epsilon_{0}=0.7 \mathrm{~K}\right)$. First, the HBT noise does not depend much on $\Gamma$, but the central dip sinks drastically as $\Gamma$ is increased, leading to a much higher contrast. The more resolved a packet is in energy, the more it decoheres and the worse the contrast.

To conclude, strong coupling between the copropagating channels accounts for a sensible loss of contrast of the HOM central dip as observed in the experiment [12]. This reduction factor strongly depends on the energy resolution of the emitted packets and is directly related to decoherence. Moreover, fast and slow modes do interfere with each other and, depending on the charge carried by the colliding excitations, produce smaller asymmetric side dips or side peaks. While these have not yet been observed, upcoming experiments with better resolution should reveal such signatures, especially when operating at lower excitation frequency, thus, accessing a wider interval of $\delta T$. This constitutes an important test, along with the expected variations in $L$ and $\Gamma$. The predicted behavior as $L$ is varied could be checked if lateral gates were added to the setup, modifying the propagation path before the QPC. Measurements with different injection energies and packet widths are already being processed. Extensions to long-range interactions [29] and to the fractional QHE are considered.

We acknowledge the support of Grants No. ANR-2010BLANC-0412, No. ANR-11-LABX-0033, No. ANR-11IDEX-0001-02, and the Mésocentre d'Aix-Marseille Université. We are grateful to P. Degiovanni, G. Fève, and F. Portier for useful discussions. 
During the process of review of the manuscript, we came aware of the work of [41], which demonstrates a new kind of electron source relevant for the present work.

*wahl@cpt.univ-mrs.fr

[1] R. H. Brown and R. Q. Twiss, Proc. R. Soc. A 242, 300 (1957).

[2] R. H. Brown and R. Q. Twiss, Proc. R. Soc. A 243, 291 (1958).

[3] C. K. Hong, Z. Y. Ou, and L. Mandel, Phys. Rev. Lett. 59, 2044 (1987).

[4] J. Beugnon, M. P. A. Jones, J. Dingjan, B. Darquié, G. Messin, A. Browaeys, and P. Grangier, Nature (London) 440, 779 (2006).

[5] J. F. Clauser, Phys. Rev. D 9, 853 (1974).

[6] G. Fève, A. Mahé, J.-M. Berroir, T. Kontos, B. Plaçais, D. C. Glatti, A. Cavanna, B. Etienne, and Y. Jin, Science 316, 1169 (2007).

[7] A. Mahé, F. D. Parmentier, E. Bocquillon, J.-M. Berroir, D. C. Glattli, T. Kontos, B. Plaçais, G. Fève, A. Cavanna, and Y. Jin, Phys. Rev. B 82, 201309 (2010).

[8] C. Leicht, P. Mirovsky, B. Kaestner, F. Hohls, V. Kashcheyevs, E. V. Kurganova, U. Zeitler, T. Weimann, K. Pierz, and H. W. Schumacher, Semicond. Sci. Technol. 26, 055010 (2011)

[9] S. Giblin, M. Kataoka, J. Fletcher, P. See, T. Janssen, J. Griffiths, G. Jones, I. Farrer, and D. Ritchie, Nat. Commun. 3, 930 (2012).

[10] S. Hermelin, S. Takada, M. Yamamoto, S. Tarucha, A. D. Wieck, L. Saminadayar, C. Bäuerle, and T. Meunier, Nature (London) 477, 435 (2011).

[11] R. P. G. McNeil, M. Kataoka, C. J. B. Ford, C. H. W. Barnes, D. Anderson, G. A. C. Jones, I. Farrer, and D. A. Ritchie, Nature (London) 477, 439 (2011).

[12] E. Bocquillon, V. Freulon, J.-M. Berroir, P. Degiovanni, B. Plaçais, A. Cavanna, Y. Jin, and G. Fève, Science 339, 1054 (2013).

[13] G. Burkard and D. Loss, Phys. Rev. Lett. 91, 087903 (2003).

[14] V. Giovannetti, D. Frustaglia, F. Taddei, and R. Fazio, Phys. Rev. B 74, 115315 (2006).

[15] M. Moskalets and M. Büttiker, Phys. Rev. B 83, 035316 (2011).

[16] S. Ol'khovskaya, J. Splettstoesser, M. Moskalets, and M. Büttiker, Phys. Rev. Lett. 101, 166802 (2008).

[17] T. Jonckheere, J. Rech, C. Wahl, and T. Martin, Phys. Rev. B 86, 125425 (2012).

[18] I. P. Levkivskyi and E. V. Sukhorukov, Phys. Rev. B 78, 045322 (2008).

[19] I. Neder, Phys. Rev. Lett. 108, 186404 (2012).

[20] E. Berg, Y. Oreg, E.-A. Kim, and F. von Oppen, Phys. Rev. Lett. 102, 236402 (2009).
[21] I. P. Levkivskyi and E. V. Sukhorukov, Phys. Rev. Lett. 109, 246806 (2012).

[22] D. L. Kovrizhin and J. T. Chalker, Phys. Rev. B 84, 085105 (2011).

[23] M. Milletarì and B. Rosenow, Phys. Rev. Lett. 111, 136807 (2013).

[24] C. Altimiras, H. le Sueur, U. Gennser, A. Cavanna, D. Mailly, and F. Pierre, Nat. Phys. 6, 34 (2009).

[25] H. le Sueur, C. Altimiras, U. Gennser, A. Cavanna, D. Mailly, and F. Pierre, Phys. Rev. Lett. 105, 056803 (2010).

[26] A. M. Lunde, S. E. Nigg, and M. Büttiker, Phys. Rev. B 81, 041311 (2010).

[27] P. Degiovanni, C. Grenier, G. Fève, C. Altimiras, H. le Sueur, and F. Pierre, Phys. Rev. B 81, 121302 (2010).

[28] J. von Delft and H. Schoeller, Ann. Phys. (Berlin) 7, 225 (1998).

[29] E. Bocquillon, V. Freulon, J.-M. Berroir, P. Degiovanni, B. Plaçais, A. Cavanna, Y. Jin, and G. Fève, Nat. Commun. 4, 1839 (2013).

[30] T. Martin, in Nanophysics: Coherence and Transport: École d'Été de Physique des Houches: Session LXXXI: 2004: Euro Summer School, Nato Advanced Study Institute, Ecole Thematique du CNRS, edited by H. Bouchiat, Y. Gefen, S. Guéron, G. Montambaux, and J. Dalibard (Elsevier, Amsterdam, 2004), p. 283.

[31] T. Martin and R. Landauer, Phys. Rev. B 45, 1742 (1992).

[32] See Supplemental Material at http://link.aps.org/ supplemental/10.1103/PhysRevLett.112.046802 for the justification of the scattering approach used to describe the QPC, a brief discussion of zero temperature results, and details on the numerical integration.

[33] C. Grenier, R. Hervé, E. Bocquillon, F. Parmentier, B. Plaçais, J.-M. Berroir, G. Fève, and P. Degiovanni, New J. Phys. 13, 093007 (2011).

[34] T. Hahn, Comput. Phys. Commun. 168, 78 (2005).

[35] P. Degiovanni, C. Grenier, and G. Fève, Phys. Rev. B 80, 241307 (2009).

[36] H. Lee and L. Levitov, arXiv:cond-mat/9312013.

[37] E. Bocquillon, F.-D. Parmentier, C. Grenier, J.-M. Berroir, P. Degiovanni, D.-C. Glattli, B. Plaçais, A. Cavanna, Y. Jin, and G. Fève, Phys. Rev. Lett. 108, 196803 (2012).

[38] G. Fève, P. Degiovanni, and T. Jolicoeur, Phys. Rev. B 77, 035308 (2008).

[39] E. Bocquillon, Ph.D. thesis, Université Paris VI, 2012.

[40] And similarly, the feature at $\delta T=-2 L u /\left(1-u^{2}\right)$ corresponds to the collision between a slow right-moving excitation and a fast left-moving one.

[41] J. Dubois, T. Jullien, F. Portier, P. Roche, A. Cavanna, Y. Jin, W. Wegscheider, P. Roulleau, and D. C. Glattli, Nature (London) 502, 659 (2013). 